\title{
Decay of a Yang-Mills Field Coupled to a Scalar Field $\star$
}

\author{
Robert T. Glassey ${ }^{1}$ and Walter A. Strauss ${ }^{2}$ \\ 1 Department of Mathematics, Indiana University, Bloomington, Indiana 47401, USA \\ 2 Department of Mathematics, Brown University, Providence, Rhode Island 02912, USA
}

\begin{abstract}
Consider a gauge field $F$ and a scalar field $\phi$ with a self-coupling $V(\phi)$ as well as the standard coupling between $F$ and $\phi$. If $0 \leqq 2 V(\phi) \leqq \phi \cdot V^{\prime}(\phi)$, there are no classical lumps. If $V(\phi)=|\phi|^{4}$ the system is conformally invariant and all the energy radiates out along the light cone.
\end{abstract}

\section{Introduction}

Consider the Lagrangian density

$$
\mathscr{L}=-\frac{1}{4} F^{2}+\frac{1}{2}(D \phi)^{2}-V(\phi)
$$

in Minkowski space, where $F$ denotes a Yang-Mills field, $\phi$ a scalar field, and $V$ a self-coupling depending only on $|\phi|^{2}$. The internal symmetry group $(5 \mathrm{5}$ is a compact Lie group, and $D$ denotes the covariant derivative.

In [1] we considered a pure Yang-Mills field with Lagrangian $F^{2}$. We proved that all the energy of a solution to the Yang-Mills equations radiates out along the light cone. We prove here the exact analogue in the case of (1) provided $V$ satisfies the inequality

$$
0 \leqq 4 V(\phi) \leqq \phi \cdot V^{\prime}(\phi) .
$$

A typical case is $V(\phi)=c|\phi|^{p}$ where $c>0, p \geqq 4$.

In case $V(\phi)=c|\phi|^{4}$, our Lagrangian $\mathscr{L}$ is invariant under the conformal group of Minkowski space (Theorem 1). This 15 dimensional Lie group generates 15 conservation laws satisfied by the solutions of the equations of motion. We derive the explicit forms of these laws in Sect. 3. In Sect. 4 we show that one of them, the First Inversional Law, implies the decay result mentioned above. For arbitrary $V$, they are no longer all conservation laws. However, under assumption (2) the extra terms in the First Inversional Law have the proper signs and we infer the same decay result.

* $\quad$ Research supported in part by NSF grants MCS 77-01340 and MCS 78-03567 
In Sect. 5 we allow the potential $V$ to include a positive mass term. Assuming only $V \geqq 0$, we deduce that certain components of the energy density are square integrable over light cone surfaces (Theorem 3). If $V$ satisfies

$$
0 \leqq 2 V(\phi) \leqq \phi \cdot V^{\prime}(\phi)
$$

then the energy within a fixed region of space is integrable in time and decays to zero as $t \rightarrow \infty$ (Theorems 4 and 5). It follows that there are no "classical lumps" if (3) holds; that is, no solutions of finite energy which are independent of time (Corollary 3).

We do not consider the question of the global existence of solutions but simply derive properties which any smooth solution would have to satisfy. For reference to related work, see [1]. In the case of a pure scalar field, the method of Sect. 5 derives originally from [2], and the conditions (2) and (3) are well-known [3].

\section{The Equations}

We begin with some notational conventions. Let $(\mathfrak{5}$ be a Lie group and $\mathfrak{g}$ its Lie algebra. We denote the Lie multiplication by $A \times B$ (rather than the usual $[A, B]$ ). Thus $A \times A=0, A \times B=-B \times A$ and the Jacobi identity takes the form

$$
A \times(B \times C)+C \times(A \times B)+B \times(C \times A)=0 .
$$

We assume $(\mathfrak{B}$ is compact. Then $\mathfrak{g}$ possesses a natural inner product which we denote by $A \cdot B$, and $(A \times B) \cdot C=A \cdot(B \times C)$. We let $|A|^{2}=A \cdot A$. In the special case when $\mathfrak{G}=\mathrm{SU}(2), \mathfrak{g}$ is three-dimensional and its elements can be regarded as vectors with $\times$ and . denoting the ordinary cross and dot products.

We write the space variables as $x=\left(x_{1}, x_{2}, x_{3}\right)$ and the time variable as $t$. We write partial derivatives as $\partial^{k}=\frac{\partial}{\partial x_{k}}(k=1,2,3)$ and $\partial^{0}=\frac{\partial}{\partial t}{ }^{1}$. We do not raise or lower indices; these are ordinary calculus derivatives. The gauge potentials $A^{\mu}$ $(\mu=0,1,2,3)$ are functions on space-time with values in $\mathfrak{g}$, as is the scalar field $\phi$. The covariant derivatives are defined by

$$
\begin{aligned}
& D^{0}=\partial^{0}-g A^{0} \times \\
& D^{k}=\partial^{k}+g A^{k} \times \quad(k=1,2,3) .
\end{aligned}
$$

We will repeatedly use the identity

$$
D^{\mu} A \cdot B+A \cdot D^{\mu} B=\partial^{\mu}(A \cdot B) \quad(\mu=0,1,2,3),
$$

where $A, B$ are $C^{1}$ vector fields. Indeed, let $\mu=1,2,3$. Then by (4.k) we have

$$
\begin{aligned}
D^{\mu} A \cdot B+A \cdot D^{\mu} B & =\left(\partial^{\mu} A+g A^{\mu} \times A\right) \cdot B+A \cdot\left(\partial^{\mu} B+g A^{\mu} \times B\right) \\
& =\partial^{\mu}(A \cdot B)+g\left(A^{\mu} \times A\right) \cdot B+g A \cdot\left(A^{\mu} \times B\right) .
\end{aligned}
$$

But $A \cdot\left(A^{\mu} \times B\right)=B \cdot\left(A \times A^{\mu}\right)$. This proves $(*)$ for $\mu=1,2,3$, and the proof for $\mu=0$ is similar.

We write the Yang-Mills field strengths as

$$
F^{\mu \nu}=\partial^{\mu} A^{v}-\partial^{v} A^{\mu}+g A^{\mu} \times A^{v} \quad(\mu, v=1,2,3)
$$

1 In [1] $\partial^{0}$ was defined as $-\frac{\partial}{\partial t}$ 
and

$$
F^{k 0}=\partial^{k} A^{0}+\partial^{0} A^{k}+g A^{k} \times A^{0} \quad(k=1,2,3)
$$

and also denote

$$
\begin{array}{lll}
E^{1}=F^{10}, & E^{2}=F^{20}, & E^{3}=F^{30}, \\
H^{1}=F^{32}, & H^{2}=F^{13}, & H^{3}=F^{21} .
\end{array}
$$

Further, we put

$$
\psi^{\mu}=D^{\mu} \phi \quad(\mu=0,1,2,3) .
$$

We consistently use $\sum_{k}, \sum_{\mu}$ to denote sums over the indices $k=1,2,3$ and $\mu=0,1,2,3$, respectively.

Let $E$ be the matrix with the columns $E^{1}, E^{2}$, and $E^{3}$, each of which belongs to g. If $\alpha=\left(\alpha_{1}, \alpha_{2}, \alpha_{3}\right)$ is an ordinary 3-vector, then $E \alpha=\sum_{k} \alpha_{k} E^{k}$. If we denote $|E|^{2}=\sum_{k}\left|E^{k}\right|^{2}$, then $|E \alpha| \leqq|E||\alpha|$. Similarly, let $H$ be the matrix with columns $H^{1}$, $H^{2}$, and $H^{3}$ and let $\Psi$ be the matrix with the columns $\psi^{1}, \psi^{2}$, and $\psi^{3}$.

Let $V_{0}$ be a real function of a real variable and define $V(\phi)=V_{0}\left(|\phi|^{2}\right)$. Let $V^{\prime}(\phi)$ denote $2 \phi V_{0}^{\prime}\left(|\phi|^{2}\right)$ where $V_{0}^{\prime}$ is the derivative of $V_{0}$.

The Lagrangian density is

$$
\mathscr{L}=\frac{1}{2} \sum_{k}\left|E^{k}\right|^{2}-\frac{1}{2} \sum_{k}\left|H^{k}\right|^{2}+\frac{1}{2}\left|D^{0} \phi\right|^{2}-\frac{1}{2} \sum_{k}\left|D^{k} \phi\right|^{2}-V(\phi) .
$$

The Euler equations for $\mathscr{L}$ take the following explicit form:

$$
\begin{aligned}
D^{0} H^{1} & =D^{3} E^{2}-D^{2} E^{3}, \\
D^{0} H^{2} & =D^{1} E^{3}-D^{3} E^{1}, \\
D^{0} H^{3} & =D^{2} E^{1}-D^{1} E^{2}, \\
D^{0} E^{1} & =D^{2} H^{3}-D^{3} H^{2}+g \psi^{1} \times \phi, \\
D^{0} E^{2} & =D^{3} H^{1}-D^{1} H^{3}+g \psi^{2} \times \phi, \\
D^{0} E^{3} & =D^{1} H^{2}-D^{2} H^{1}+g \psi^{3} \times \phi, \\
\sum_{k} D^{k} H^{k} & =0, \\
\sum_{k} D^{k} E^{k} & =g \psi^{0} \times \phi, \\
D^{0} \psi^{0}-\sum_{k} D^{k} \psi^{k} & =-V^{\prime}(\phi), \\
D^{0} \psi^{j}-D^{j} \psi^{0} & =g E^{j} \times \varphi \\
D^{1} \psi^{2}-D^{2} \psi^{1} & =-g H^{3} \times \phi, \quad(j=1,2,3), \\
D^{2} \psi^{3}-D^{3} \psi^{2} & =-g H^{1} \times \phi, \\
D^{3} \psi^{1}-D^{1} \psi^{3} & =-g H^{2} \times \varphi .
\end{aligned}
$$


Derivation of Equations (11)-(15). Actually, only Eq. (12), (13.E), and (14.0) result directly from $\mathscr{L}$ as equations of motion. The rest of the Eq. (11)-(15) are "constraint" equations which follow from the definitions of $F^{\mu v}$ and $\psi^{\mu}$, as we now demonstrate.

The Euler equations of (10) have the form

$$
\frac{\partial \mathscr{L}}{\partial \phi}=\sum_{\mu} \partial^{\mu} \frac{\partial \mathscr{L}}{\partial\left(\partial^{\mu} \phi\right)}
$$

and

$$
\frac{\partial \mathscr{L}}{\partial A^{\mu}}=\sum_{v} \partial^{v} \frac{\partial \mathscr{L}}{\partial\left(\partial^{v} A^{\mu}\right)} \quad(\mu=0,1,2,3) .
$$

The derivatives of $\mathscr{L}$ have values in $\mathfrak{g}$. Thus for instance,

$$
\frac{\partial \mathscr{L}}{\partial \phi} \cdot \tilde{\phi}=\left.\frac{d}{d \varepsilon} \mathscr{L}(\phi+\varepsilon \tilde{\phi})\right|_{\varepsilon=0} .
$$

In order to evaluate (16) and (17) it is convenient to note that

$$
\text { (i) } \frac{\partial}{\partial A} \frac{1}{2}|A+(B \times C)|^{2}=A+(B \times C)
$$

and

ii) $\frac{\partial}{\partial B} \frac{1}{2}|A+(B \times C)|^{2}=C \times\{A+(B \times C)\}$.

Indeed, i) is obvious and ii) follows from

$$
\begin{aligned}
\frac{d}{d \varepsilon} \frac{1}{2}|A+((B+\varepsilon \tilde{B}) \times C)|^{2} & =\tilde{B} \times C \cdot\{A+(B \times C)\} \\
& =\tilde{B} \cdot C \times\{A+(B \times C)\} \text { at } \varepsilon=0 .
\end{aligned}
$$

We now evaluate (16).

$$
\begin{aligned}
\frac{\partial \mathscr{L}}{\partial \phi} & =\frac{1}{2} \frac{\partial}{\partial \phi}\left\{\left|\partial^{0} \phi-g A^{0} \times \phi\right|^{2}-\sum_{k}\left|\partial^{k} \phi+g A^{k} \times \phi\right|^{2}-2 V(\phi)\right\} \\
& =g A^{0} \times \psi^{0}+g \sum_{k} A^{k} \times \psi^{k}-V^{\prime}(\phi) .
\end{aligned}
$$

Clearly $\frac{\partial \mathscr{L}}{\partial\left(\partial^{0} \phi\right)}=\psi^{0}$ and $\frac{\partial \mathscr{L}}{\partial\left(\partial^{j} \phi\right)}=-\psi^{j}(j=1,2,3)$ by i).

Hence (16) implies

$$
\partial^{0} \psi^{0}-\sum_{k} \partial^{k} \psi^{k}=g A^{0} \times \psi^{0}+g \sum_{k} A^{k} \times \psi^{k}-V^{\prime}(\phi)
$$

which is precisely (14.0) in view of (4.0) and (4.k).

Next, consider (17.0). Then by ii) we find

$$
\begin{aligned}
\frac{\partial \mathscr{L}}{\partial A^{0}} & =\frac{1}{2} \frac{\partial}{\partial A^{0}}\left\{\sum_{k}\left|\partial^{k} A^{0}+\partial^{0} A^{k}-g A^{0} \times A^{k}\right|^{2}+\left|\partial^{0} \phi-g A^{0} \times \phi\right|^{2}\right\} \\
& =-g \sum_{k} A^{k} \times E^{k}-g \phi \times \psi^{0} .
\end{aligned}
$$


$\mathscr{L}$ does not depend on $\partial^{0} A^{0}$ while

$$
\partial \mathscr{L} / \partial\left(\partial^{j} A^{0}\right)=E^{j} \quad \text { by i) . }
$$

Therefore (17.0) implies

$$
\sum_{k} \partial^{k} E^{k}=-g \sum_{k} A^{k} \times E^{k}-g \phi \times \psi^{0},
$$

which is the same as (13.E).

We now consider $(17 . k)$ for $k=1$ say. We have

$$
\begin{aligned}
\frac{\partial \mathscr{L}}{\partial A^{1}}= & \frac{1}{2} \frac{\partial}{\partial A^{1}}\left\{\left|\partial^{1} A^{0}+\partial^{0} A^{1}+g A^{1} \times A^{0}\right|^{2}-\left|\partial^{1} A^{3}-\partial^{3} A^{1}+g A^{1} \times A^{3}\right|^{2}\right. \\
& \left.-\left|\partial^{2} A^{1}-\partial^{1} A^{2}-g A^{1} \times A^{2}\right|^{2}-\left|\partial^{1} \phi+g A^{1} \times \phi\right|^{2}\right\} .
\end{aligned}
$$

By ii) we have

$$
\begin{aligned}
\frac{\partial \mathscr{L}}{\partial A^{1}} & =g A^{0} \times E^{1}-g A^{3} \times F^{13}+g A^{2} \times F^{21}-g \phi \times \psi^{1} \\
& =g A^{0} \times E^{1}-g A^{3} \times H^{2}+g A^{2} \times H^{3}-g \phi \times \psi^{1}
\end{aligned}
$$

Furthermore, by i),

$$
\frac{\partial \mathscr{L}}{\partial\left(\partial^{0} A^{1}\right)}=E^{1}, \quad \frac{\partial \mathscr{L}}{\partial\left(\partial^{2} A^{1}\right)}=-F^{21}=-H^{3}, \quad \text { and } \quad \frac{\partial \mathscr{L}}{\partial\left(\partial^{3} A^{1}\right)}=F^{13}=H^{2} .
$$

Since $\mathscr{L}$ does not depend on $\partial^{1} A^{1},(17.1)$ yields

$\partial^{0} E^{1}-\partial^{2} H^{3}+\partial^{3} H^{2}=g A^{0} \times E^{1}-g A^{3} \times H^{2}+g A^{2} \times H^{3}-g \phi \times \psi^{1}$

which is the same as (12.1).

Next we indicate the derivation of the "constraint" equations. In each case below the Jacobi identity is used.

Consider (11.1). We have

$$
\begin{aligned}
D^{0} H^{1}= & D^{0} F^{32}=\left(\partial^{0}-g A^{0} \times\right)\left(\partial^{3} A^{2}-\partial^{2} A^{3}+g A^{3} \times A^{2}\right) \\
= & \partial^{3} \partial^{0} A^{2}-\partial^{2} \partial^{0} A^{3}+g \partial^{0}\left(A^{3} \times A^{2}\right)-g A^{0} \times \partial^{3} A^{2} \\
& +g A^{0} \times \partial^{2} A^{3}-g^{2} A^{0} \times\left(A^{3} \times A^{2}\right) .
\end{aligned}
$$

In the second line replace

$$
\partial^{0} A^{2} \quad \text { by } \quad E^{2}-\partial^{2} A^{0}-g A^{2} \times A^{0}
$$

and

$$
\partial^{0} A^{3} \text { by } E^{3}-\partial^{3} A^{0}-g A^{3} \times A^{0} .
$$

Hence

$$
\begin{aligned}
D^{0} H^{1}= & \partial^{3} E^{2}-\partial^{2} E^{3}-g \partial^{3}\left(A^{2} \times A^{0}\right)+g \partial^{2}\left(A^{3} \times A^{0}\right) \\
& +g \partial^{0}\left(A^{3} \times A^{2}\right)-g A^{0} \times \partial^{3} A^{2}+g A^{0} \times \partial^{2} A^{3}-g^{2} A^{0} \times\left(A^{3} \times A^{2}\right) .
\end{aligned}
$$


Now we write the first two terms here as

$$
\partial^{3} E^{2}-\partial^{2} E^{3}=D^{3} E^{2}-g A^{3} \times E^{2}-D^{2} E^{3}+g A^{2} \times E^{3}
$$

and evaluate the cross products $A^{3} \times E^{2}, A^{2} \times E^{3}$ using (6) and (7). Then in the expression for $D^{0} H^{1}$ above, the quadratic terms cancel pairwise, and the three cubic terms sum to zero by the Jacobi identity. This establishes (11.1); (11.2) and (11.3) follow from cyclic permutation.

Consider next (13.H). By (4.1) we have

$$
\begin{aligned}
D^{1} H^{1}= & \partial^{1} H^{1}+g A^{1} \times H^{1}=\partial^{1} F^{32}+g A^{1} \times F^{32} \\
= & \partial^{1}\left(\partial^{3} A^{2}-\partial^{2} A^{3}+g A^{3} \times A^{2}\right) \\
& +g A^{1} \times \partial^{3} A^{2}-g A^{1} \times \partial^{2} A^{3}+g^{2} A^{1} \times\left(A^{3} \times A^{2}\right) .
\end{aligned}
$$

$D^{2} H^{2}$ and $D^{3} H^{3}$ can be evaluated from this by cyclic permutation. When we form the sum $\sum_{k} D^{k} H^{k}$, the second-derivative terms cancel pairwise, as do the quadratic terms. Again the Jacobi identity shows that the three cubic terms vanish upon summation. Therefore $\sum_{k} D^{k} H^{k}=0$ which is $(13 . H)$.

To establish (14.j), we write (for $j=1,2,3$ )

$$
\begin{aligned}
D^{0} \psi^{j}-D^{j} \psi^{0} & =\partial^{0} \psi^{j}-\partial^{j} \psi^{0}-g A^{0} \times \psi^{j}-g A^{j} \times \psi^{0} \\
& =\partial^{0}\left(\partial^{j} \phi+g A^{j} \times \phi\right)-\partial^{j}\left(\partial^{0} \phi-g A^{0} \times \phi\right)-g A^{0} \times \psi^{j}-g A^{j} \times \psi^{0} \\
& =g\left(\partial^{0} A^{j}+\partial^{j} A^{0}\right) \times \phi+g A^{j} \times\left(\partial^{0} \phi-\psi^{0}\right)+g A^{0} \times\left(\partial^{j} \phi-\psi^{j}\right) .
\end{aligned}
$$

Using (7), (9.0), and (9.j), this reduces to

$$
g\left(E^{j}-g A^{j} \times A^{0}\right) \times \phi+g^{2} A^{j} \times\left(A^{0} \times \phi\right)-g^{2} A^{0} \times\left(A^{j} \times \phi\right)=g E^{j} \times \phi .
$$

Finally, we verify (15.1). We have

$$
\begin{aligned}
D^{1} \psi^{2}-D^{2} \psi^{1}= & \partial^{1} \psi^{2}+g A^{1} \times \psi^{2}-\partial^{2} \psi^{1}-g A^{2} \times \psi^{1} \\
= & \partial^{1}\left(\partial^{2} \phi+g A^{2} \times \phi\right)-\partial^{2}\left(\partial^{1} \phi+g A^{1} \times \phi\right) \\
& +g A^{1} \times\left(\partial^{2} \phi+g A^{2} \times \phi\right)-g A^{2} \times\left(\partial^{1} \phi+g A^{1} \times \phi\right) .
\end{aligned}
$$

Six terms cancel pairwise and we get

$$
g\left(\partial^{1} A^{2}-\partial^{2} A^{1}\right) \times \phi+g^{2} A^{1} \times\left(A^{2} \times \phi\right)-g^{2} A^{2} \times\left(A^{1} \times \phi\right) .
$$

We substitute

$$
\partial^{1} A^{2}-\partial^{2} A^{1}=-H^{3}-g A^{1} \times A^{2}
$$

and use the Jacobi identity to obtain (15.1). This completes the derivation of the full set of equations of motion (11)-(15). 


\section{The Conservation Laws}

The energy is obtained as follows. Multiply (11.j) by $H^{j}$ and $(12 . j)$ by $E^{j}(j=1,2,3)$ and add the resulting six equations. Using $(*)$ we get the identity

$$
\partial^{0} e_{Y M}=\sum_{k}\left[\partial^{k} p_{Y M}^{k}+g \psi^{k} \times \phi \cdot E^{k}\right],
$$

where $e_{Y M}=\frac{1}{2}\left(|E|^{2}+|H|^{2}\right)$ and

$$
\begin{aligned}
& p_{Y M}^{1}=H^{2} \cdot E^{3}-E^{2} \cdot H^{3} \\
& p_{Y M}^{2}=H^{3} \cdot E^{1}-E^{3} \cdot H^{1} \\
& p_{Y M}^{3}=H^{1} \cdot E^{2}-E^{1} \cdot H^{2} .
\end{aligned}
$$

Next we multiply $(14 . \mu)$ by $\psi^{\mu}(\mu=0,1,2,3)$ and add the resulting four equations to obtain

$$
\begin{aligned}
\frac{1}{2} \partial^{0} \sum_{\mu}\left|\psi^{\mu}\right|^{2} & =-V^{\prime}(\phi) \cdot \psi^{0}+\sum_{k} \partial^{k}\left(\psi^{0} \cdot \psi^{k}\right)+g \sum_{k} E^{k} \times \phi \cdot \psi^{k} \\
& =-\partial^{0} V(\phi)+\sum_{k}\left[\partial^{k}\left(\psi^{0} \cdot \psi^{k}\right)+g E^{k} \times \phi \cdot \psi^{k}\right] .
\end{aligned}
$$

Adding (18) and (19) we get the Energy Conservation Law

$$
\partial^{0} e=\sum_{k} \partial^{k} p^{k}
$$

where

$$
e=\frac{1}{2}\left(|E|^{2}+|H|^{2}+\sum_{\mu}\left|\psi^{\mu}\right|^{2}\right)+V(\phi)
$$

and

$$
p^{k}=p_{Y M}^{k}+\psi^{0} \cdot \psi^{k} \quad(k=1,2,3) .
$$

Next we calculate the momenta. Consider

$$
p^{1}=H^{2} \cdot E^{3}-H^{3} \cdot E^{2}+\psi^{0} \cdot \psi^{1} .
$$

Using $(*)$ we have

$$
\partial^{0} p^{1}=I+I I,
$$

where

$$
\begin{aligned}
I & =D^{0} H^{2} \cdot E^{3}+H^{2} \cdot D^{0} E^{3}-D^{0} H^{3} \cdot E^{2}-H^{3} \cdot D^{0} E^{2}, \\
I I & =D^{0} \psi^{0} \cdot \psi^{1}+\psi^{0} \cdot D^{0} \psi^{1} .
\end{aligned}
$$

Using (11), (12), and (14) we have

$$
\begin{aligned}
I= & \left(D^{1} E^{3}-D^{3} E^{1}\right) \cdot E^{3}+H^{2} \cdot\left(D^{1} H^{2}-D^{2} H^{1}+g \psi^{3} \times \phi\right) \\
& -\left(D^{2} E^{1}-D^{1} E^{2}\right) \cdot E^{2}-H^{3} \cdot\left(D^{3} H^{1}-D^{1} H^{3}+g \psi^{2} \times \phi\right) ; \\
I I= & \left(\sum_{k} D^{k} \psi^{k}-V^{\prime}(\phi)\right) \cdot \psi^{1}+\psi^{0} \cdot\left(D^{1} \psi^{0}+g E^{1} \times \phi\right) .
\end{aligned}
$$


Using $(*)$ we can write these as

$$
\begin{aligned}
I= & \frac{1}{2} \partial^{1}\left(\left|E^{2}\right|^{2}+\left|E^{3}\right|^{2}+\left|H^{2}\right|^{2}+\left|H^{3}\right|^{2}\right) \\
& -\partial^{2}\left(E^{1} \cdot E^{2}+H^{1} \cdot H^{2}\right)-\partial^{3}\left(E^{1} \cdot E^{3}+H^{1} \cdot H^{3}\right) \\
& +E^{1} \cdot\left(D^{2} E^{2}+D^{3} E^{3}\right)+\left(D^{2} H^{2}+D^{3} H^{3}\right) \cdot H^{1} \\
& +g H^{2} \cdot \psi^{3} \times \phi-g H^{3} \cdot \psi^{2} \times \phi ; \\
I I= & \frac{1}{2} \partial^{1}\left(\left|\psi^{0}\right|^{2}+\left|\psi^{1}\right|^{2}-2 V(\phi)\right)+\partial^{2}\left(\psi^{1} \cdot \psi^{2}\right)+\partial^{3}\left(\psi^{1} \cdot \psi^{3}\right) \\
& -\psi^{2} \cdot D^{2} \psi^{1}-\psi^{3} \cdot D^{3} \psi^{1}+g \psi^{0} \cdot E^{1} \times \phi .
\end{aligned}
$$

The third line of $I$ becomes, by (13.E),

$$
-\frac{1}{2} \partial^{1}\left(\left|E^{1}\right|^{2}+\left|H^{1}\right|^{2}\right)+g E^{1} \cdot \psi^{0} \times \phi .
$$

By (15), the second line of $I I$ becomes

$$
-\frac{1}{2} \partial^{1}\left(\left|\psi^{2}\right|^{2}+\left|\psi^{3}\right|^{2}\right)-g \psi^{2} \cdot H^{3} \times \phi+g \psi^{3} \cdot H^{2} \times \phi+g \psi^{0} \cdot E^{1} \times \phi .
$$

All the cubic terms drop out and we obtain (for $j=1$ ) the Momentum Conservation Law

$$
\partial^{0} p^{j}=\partial^{j} f+\sum_{k} \partial^{k} q^{j k}
$$

where

$$
f=e-|\Psi|^{2}-2 V(\phi)
$$

and

$$
q^{j k}=-E^{j} \cdot E^{k}-H^{j} \cdot H^{k}+\psi^{j} \cdot \psi^{k} .
$$

The equations for $j=2,3$ are obtained in exactly the same way.

The other eleven identities are derivable from (20) and (23.j). They are as follows :

$$
\begin{aligned}
& \partial^{0}\left(x_{j} e+t p^{j}\right)=\partial^{j}(t f)+\sum_{k} \partial^{k}\left(x_{j} p^{k}+t q^{j k}\right)(j=1,2,3), \\
& \partial^{0}\left(x_{2} p^{1}-x_{1} p^{2}\right)=\partial^{1}\left(x_{2} f\right)-\partial^{2}\left(x_{1} f\right)+\sum_{k} \partial^{k}\left(x_{2} q^{1 k}-x_{1} q^{2 k}\right),
\end{aligned}
$$

and two similar laws obtained by cyclic permutation of indices.

$$
\begin{aligned}
& \partial^{0}\left(t e+\sum_{k} x_{k} p^{k}+\psi^{0} \cdot \phi\right)+V^{\prime}(\phi) \cdot \phi-4 V(\phi) \\
& \quad=\sum_{k} \partial^{k}\left(t p^{k}+x_{k} f+\sum_{j} x_{j} q^{j k}+\psi^{k} \cdot \phi\right), \\
& \partial^{0}\left\{\left(t^{2}+r^{2}\right) e+2 t \sum_{k} x_{k} p^{k}+2 t \psi^{0} \cdot \varphi-|\phi|^{2}\right\}+2 t\left\{V^{\prime}(\phi) \cdot \phi-4 V(\phi)\right\} \\
& \quad=\sum_{k} \partial^{k}\left\{\left(t^{2}+r^{2}\right) p^{k}+2 t\left(x_{k} f+\sum_{j} x_{j} q^{j k}+\psi^{k} \cdot \phi\right)\right\}, \\
& \partial^{0}\left\{t x_{j} e+\frac{1}{2}\left(t^{2}-r^{2}\right) p^{j}+x_{j} \sum_{k} x_{k} p^{k}+x_{j} \psi^{0} \cdot \phi\right\}+x_{j}\left\{\phi \cdot V^{\prime}(\phi)-4 V(\phi)\right\} \\
& \quad=\partial^{j}\left\{\frac{t^{2}-r^{2}}{2} f\right\}+\sum_{k} \partial^{k}\left[t x_{j} e-x_{j} \phi \cdot \psi^{k}+\frac{t^{2}-r^{2}}{2} q^{j k}+x_{j} x_{k} f+x_{j} \sum_{m} x_{m} q^{m k}\right] \\
& \quad(j=1,2,3) .
\end{aligned}
$$


Theorem 1. If $V(\phi)=c|\phi|^{4}$, the system is invariant under the conformal group.

Indeed, $4 V(\phi)=\phi \cdot V^{\prime}(\phi)$, so that all 15 identities are exact conservation laws. These are the conservation laws which follow from the invariance via Noether's Theorem. We omit the direct proof of the invariance.

As an example we present a detailed derivation of the First Inversional Law (27). Multiply (20) by $r^{2}+t^{2}$ and $(23 . j)$ by $2 t x_{j}$. The sum of the resulting four equations can be written as

$$
\begin{aligned}
\partial^{0} & {\left[\left(r^{2}+t^{2}\right) e+2 t \sum_{j} x_{j} p^{j}\right] } \\
& =\sum_{k} \partial^{k}\left[\left(r^{2}+t^{2}\right) p^{k}+2 t x_{k} f+2 t \sum_{j} x_{j} q^{j k}\right]+2 t\left[e-3 f-\sum_{k} q^{k k}\right] .
\end{aligned}
$$

The last expression is

$$
e-3 f-\sum_{k} q^{k k}=-2 e+|E|^{2}+|H|^{2}+2|\Psi|^{2}+6 V(\phi)=-\left|\psi^{0}\right|^{2}+|\Psi|^{2}+4 V(\phi) .
$$

Now multiply (14.0) by $\phi$ and make use of $(*)$ to obtain

$$
\partial^{0}\left(\psi^{0} \cdot \phi\right)-\psi^{0} \cdot D^{0} \phi-\sum_{k}\left(\partial^{k}\left(\psi^{k} \cdot \phi\right)-\psi^{k} \cdot D^{k} \phi\right)=-\phi \cdot V^{\prime}(\phi) .
$$

By $(9 . \mu)$ this can be written as

$$
\partial^{0}\left(\psi^{0} \cdot \phi\right)+V^{\prime}(\phi) \cdot \phi=\left|\psi^{0}\right|^{2}-|\Psi|^{2}+\sum_{k} \partial^{k}\left(\psi^{k} \cdot \phi\right)
$$

Multiplying this by $2 t$, we get

$$
\partial^{0}\left[\left(2 t \psi^{0}-\phi\right) \cdot \phi\right]+2 t \phi \cdot V^{\prime}(\phi)=2 t\left(\left|\psi^{0}\right|^{2}-|\Psi|^{2}\right)+\sum_{k} \partial^{k}\left(2 t \psi^{k} \cdot \phi\right) .
$$

When we add this result to (29), we obtain (27).

\section{Asymptotics in the Case of Zero Mass}

Theorem 2. Assume that $V$ satisfies the inequalities

$$
0 \leqq 4 V(s) \leqq s \cdot V^{\prime}(s)
$$

Let $R>0$ and $0<\varepsilon \leqq 1$. Then as $t \rightarrow \infty$,

$$
\int_{|x|<R+(1-\varepsilon) t} e d x=O\left(t^{-2}\right),
$$

where $e$ is the energy density (21). (This is valid provided $\int r^{2} e d x<\infty$ at all times.) Proof. We integrate (27) over all space. Assume that the solution is smooth and satisfies $\int r^{2} e d x<\infty$ at any time. Then the right side vanishes. We obtain

$$
\int\left\{\left(r^{2}+t^{2}\right) e+2 t \sum_{k} x_{k} p^{k}+2 t \phi \cdot \psi^{0}-\phi \cdot \phi\right\} d x \leqq \text { const }=C .
$$

We break this integrand into two parts, the part involving the Yang-Mills field $I_{Y M}$ and the part involving the scalar field $I_{s}$. Let $\omega=\frac{x}{r}$ and introduce unit vectors $\alpha$ 
and $\beta$ so that $\alpha, \beta, \omega$ form an orthonormal basis for $\mathbb{R}^{3}$ with $\alpha \times \beta=\omega$. By the orthonormal property of the basis vectors we have

$$
|E|^{2}=|E \alpha|^{2}+|E \beta|^{2}+|E \omega|^{2}
$$

and a similar identity with $E$ replaced by $H$. Then, as we showed in [1],

$$
e_{Y M} \pm \sum_{k} \omega_{k} p_{Y M}^{k}=\frac{1}{2}\left(|E \omega|^{2}+|H \omega|^{2}+|E \alpha \mp H \beta|^{2}+|E \beta \pm H \alpha|^{2}\right) .
$$

In particular, $\left|\sum_{k} \omega_{k} p_{Y M}^{k}\right| \leqq e_{Y M}$ and so

$$
I_{Y M} \geqq(t-r)^{2} e_{Y M} \text {. }
$$

We now express $I_{s}$ as a sum of squares. First we denote $\chi^{k}=\psi^{k}+\frac{x_{k}}{r^{2}} \phi$ and define $\Xi$ to be the matrix with columns $\chi^{1}, \chi^{2}, \chi^{3}$. Using $(9 . k)$ we have

$$
|\Xi|^{2}=|\Psi|^{2}+\frac{1}{r^{2}} \partial^{r}\left(r|\phi|^{2}\right)
$$

where $r=|x|$ and $r \partial^{r}=\sum_{k} x_{k} \partial^{k}$. Hence

$$
\begin{aligned}
I_{s} & =\frac{t^{2}+r^{2}}{2}\left(\left|\psi^{0}\right|^{2}+|\Psi|^{2}+2 V(\phi)\right)+2 t \psi^{0} \cdot(\Psi x+\phi)-|\phi|^{2} \\
& =I_{s}^{*}-\frac{1}{2 r^{2}} \partial^{r}\left\{\left(t^{2}+r^{2}\right) r|\phi|^{2}\right\},
\end{aligned}
$$

where

$$
\begin{aligned}
I_{s}^{*} & =\frac{t^{2}+r^{2}}{2}\left(\left|\psi^{0}\right|^{2}+|\Xi|^{2}+2 V(\phi)\right)+2 t \psi^{0} \cdot \Xi x \\
& \geqq \frac{(t-r)^{2}}{2}\left(\left|\psi^{0}\right|^{2}+|\Xi|^{2}+2 V(\phi)\right) \geqq 0 .
\end{aligned}
$$

Hence for any subset $B$ of space we have

$$
\int_{B}\left(I_{Y M}+I_{s}^{*}\right) d x \leqq \int\left(I_{Y M}+I_{s}\right) d x \leqq C .
$$

Choosing $B=\{x:|x|<R+(1-\varepsilon) t\}$ we obtain from (32) and (35)

$$
(\varepsilon t-R)^{2} \int_{B}\left\{e_{Y M}+\frac{1}{2}\left|\psi^{0}\right|^{2}+\frac{1}{2}|\Xi|^{2}+V(\phi)\right\} d x \leqq C
$$

for $t>R \varepsilon^{-1}$. Finally, using (33) again we have

$$
\int_{B}|\Psi|^{2} d x \leqq \int_{B}|\Xi|^{2} d x \leqq 2 C(\varepsilon t-R)^{-2}
$$

for $t>R \varepsilon^{-1}$. This completes the proof.

The following two corollaries are proved exactly as in [1]. 
Corollary 1. Assume that any finite-energy solution ( a solution with $\int e d x<\infty$ ) can be approximated by cut-off solutions in the energy norm, uniformly in time (see [1]). Then for any finite energy solution and for each $R>0$ and $0<\varepsilon \leqq 1$, we have

$$
\lim _{t \rightarrow \infty} \int_{|x|<R+(1-\varepsilon) t} e d x=0 .
$$

Corollary 2. Under the same assumptions, there is no finite energy solution of the form

$$
E=E(x-c t), \quad H=H(x-b t), \quad \phi=\phi(x-a t),
$$

where $a, b, c$ are constant vectors of norm less than one, except the trivial solution

$$
E=H=0, \quad \phi=\text { const }, \quad V(\phi)=0 .
$$

Remark. By (31) and (34), the integral over all space of certain of the components of $e$ is $O\left(t^{-2}\right)$.

\section{Estimates in Case the Scalar Field Has Positive Mass}

We begin by showing that certain components of the fields are square integrable over light cones.

Theorem 3. For any finite energy solution,

$$
\begin{aligned}
& \int_{|x|=t}\left\{2 V(\phi)+\left|\psi^{0}+\Psi \omega\right|^{2}+|\Psi \alpha|^{2}+|\Psi \beta|^{2}\right. \\
& \left.\quad+|E \omega|^{2}+|H \omega|^{2}+|E \alpha-H \beta|^{2}+|E \beta+H \alpha|^{2}\right\} d S \leqq \mathrm{const},
\end{aligned}
$$

where $d S$ is the usual surface measure on $\{|x|=t\}$. (The notation of Theorem 2 remains in effect.) If $V \geqq 0$, each term is positive and therefore integrable on the cone.

Proof. We integrate the energy conservation law (20) over the 4-dimensional region $\{|x|<t<T\}$ and then let $T \rightarrow \infty$ to obtain

$$
2 \int_{|x|=t}\left(e+\sum_{k} \omega_{k} p^{k}\right) d S \leqq 2 \sqrt{2} \int e d x=\text { const }
$$

where $\omega_{k}=\frac{x_{k}}{r}$. The Yang-Mills terms in the integrand are written as in (31). The other terms are $2 V(\phi)$ and

$$
\left|\psi^{0}\right|^{2}+|\Psi|^{2}+2 \psi^{0} \cdot \Psi \omega=\left|\psi^{0}+\Psi \omega\right|^{2}+|\Psi \alpha|^{2}+|\Psi \beta|^{2} .
$$

This proves Theorem 3 .

Next we turn to an investigation of the asymptotic behavior in case $V(\phi)$ includes a mass term. Typically one envisions

$$
V(\phi)=m_{0}^{2}|\phi|^{2}+c|\phi|^{p+1}\left(m_{0}>0, c>0, p>1\right) .
$$

For this purpose, assume $\phi \cdot V^{\prime}(\phi) \geqq 2 V(\phi) \geqq 0$. We employ the summation convention $(j, k=1,2,3)$ for notational simplicity. Multiply (23.j) by a function $2 l_{j}(x)(j=1,2,3)$ and sum on $j$ to get

$$
2 \partial^{0}\left(l_{j} p^{j}\right)+2\left(\partial^{j} l_{j}\right) f+2 \partial^{k} l_{j} \cdot q^{j k}=2 \partial^{j}\left(l_{j} f\right)+2 \partial^{k}\left(l_{j} q^{j k}\right) .
$$


Let $m=\partial^{J} l_{j}$. Multiply (30) by $m$ to get

$$
\begin{aligned}
& \partial^{0}\left(m \psi^{0} \cdot \phi\right)+m\left(|\Psi|^{2}-\left|\psi^{0}\right|^{2}+V^{\prime}(\phi) \cdot \phi\right) \\
& \quad=\partial^{k}\left(m \psi^{k} \cdot \phi\right)-\left(\partial^{k} m\right) \psi^{k} \cdot \phi \\
& \quad=\partial^{k}\left(m \psi^{k} \cdot \phi-\frac{1}{2}\left(\partial^{k} m\right)|\phi|^{2}\right)+\frac{1}{2}\left(\partial^{k} \partial^{k} m\right)|\phi|^{2} .
\end{aligned}
$$

Adding these two equations we find

$$
\begin{aligned}
\partial^{0}\left\{2 l_{j} p^{j}+m \psi^{0} \cdot \phi\right\} & \\
& +2 \partial^{k} l_{j} \cdot q^{j k}+m\left(2 f+|\Psi|^{2}-\left|\psi^{0}\right|^{2}+V^{\prime}(\phi) \cdot \phi\right)-\frac{1}{2}\left(\partial^{k} \partial^{k} m\right)|\phi|^{2} \\
= & \partial^{k}\left\{2 l_{k} f+2 l_{j} q^{j k}+m \psi^{k} \cdot \phi-\frac{1}{2}\left(\partial^{k} m\right)|\phi|^{2}\right\} .
\end{aligned}
$$

We write (36) in the form $\partial^{0} X+Z=\partial^{k} Y^{k}$. We evaluate $Z$ by substituting the expressions for $f$ and $q^{j k}$ :

$$
\begin{aligned}
Z= & \left(m \delta_{j k}-2 \partial^{k} l_{j}\right)\left(E^{j} \cdot E^{k}+H^{j} \cdot H^{k}\right)+2\left(\partial^{k} l_{j}\right) \psi^{j} \cdot \psi^{k} \\
& +m\left(\phi \cdot V^{\prime}(\phi)-2 V(\phi)\right)-\frac{1}{2}\left(\partial^{k} \partial^{k} m\right)|\phi|^{2} .
\end{aligned}
$$

In (36),

$$
X=2 l_{j} p^{j}+m \psi^{0} \cdot \phi=2 l_{j} p_{Y M}^{j}+2 \psi^{0} \cdot\left(l_{j} \psi^{j}+\frac{m}{2} \phi\right) .
$$

Hence

$$
|X| \leqq 2\left|l_{j} p_{Y M}^{j}\right|+\left|\psi^{0}\right|^{2}+\left|l_{j} \psi^{j}+\frac{m}{2} \phi\right|^{2} .
$$

The last term here is

$$
\begin{aligned}
\left|l_{j} \psi^{j}+\frac{m}{2} \phi\right|^{2} & =\left|l_{j} \psi^{j}\right|^{2}+\frac{1}{2} m l_{j} \partial^{j}\left(|\phi|^{2}\right)+\frac{m^{2}}{4}|\phi|^{2} \\
& =\left|l_{j} \psi^{j}\right|^{2}+\partial^{j}\left(\frac{1}{2} l_{j} m|\phi|^{2}\right)-\frac{1}{2}\left(l_{j} \partial^{j} m+\frac{1}{2} m^{2}\right)|\phi|^{2} .
\end{aligned}
$$

Now we choose $l_{j}(x)=\frac{x_{j}}{r}$. Then

$$
\partial^{k} l_{j}=\delta_{j k} / r-x_{j} x_{k} / r^{3} \quad \text { and } \quad m=\partial^{j} l_{j}=\frac{2}{r} .
$$

Therefore $m \delta_{j k}-2 \partial^{k} l_{j}=2 x_{j} x_{k} / r^{3}$,

$$
l_{j} \partial^{j} m+\frac{1}{2} m^{2}=0 \text { and } \partial^{k} \partial^{k} m=0 \text { for } x \neq 0 .
$$

It follows from (37) and (38) that

$$
|X| \leqq 2 e_{Y M}+\left|\psi^{0}\right|^{2}+|\Psi|^{2}+\partial^{j}\left(\frac{1}{2} l_{j} m|\phi|^{2}\right) .
$$

Hence $\int|X| d x$ is bounded by twice the energy. Now we integrate (36) over the exterior of a small sphere $\{|x|>\varepsilon\}$ and then let $\varepsilon \rightarrow 0$. On the right side of the 
resulting equation, the terms in (36) of the form $\partial^{k} Y^{k}$ drop out except for the last term, which gives

$$
\begin{gathered}
-\frac{1}{2} \int_{|x|>\varepsilon} \partial^{k}\left\{\left(\partial^{k} m\right)|\phi|^{2}\right\} d x=\int_{|x|>\varepsilon} \partial^{k}\left\{\frac{x_{k}}{r^{3}}|\phi|^{2}\right\} d x \\
=-\frac{1}{\varepsilon^{2}} \int_{|x|=\varepsilon}|\phi(x, t)|^{2} d S_{x} \rightarrow-4 \pi|\phi(0, t)|^{2} \leqq 0
\end{gathered}
$$

as $\varepsilon \rightarrow 0$. On the left side we have the integral of

$$
Z=\frac{2}{r}\left\{\left|E_{r}\right|^{2}+\left|H_{r}\right|^{2}+|\Psi|^{2}-\left|\Psi_{r}\right|^{2}+V^{\prime}(\phi) \cdot \phi-2 V(\phi)\right\},
$$

where we have denoted

$$
E_{r}=\frac{1}{r} E x, \quad H_{r}=\frac{1}{r} H x, \quad \Psi_{r}=\frac{1}{r} \Psi x .
$$

Thus we have proved that

$$
\iint_{-\infty}^{\infty} Z d x d t+4 \pi \int_{-\infty}^{\infty}|\phi(0, t)|^{2} d t \leqq 4 \int e d x=4 e_{0}
$$

Theorem 4. Assume $\phi \cdot V^{\prime}(\phi) \geqq 2 V(\phi) \geqq 0$. Consider a smooth solution of finite energy. Then

$$
\begin{aligned}
& \iint\left(\left|E_{r}\right|^{2}+\left|H_{r}\right|^{2}\right) \frac{1}{r} d x d t<\infty, \\
& \iint\left(|\Psi|^{2}-\left|\Psi_{r}\right|^{2}\right) \frac{1}{r} d x d t<\infty, \\
& \iint\left(\phi \cdot V^{\prime}(\phi)-2 V(\phi)\right) \frac{1}{r} d x d t<\infty .
\end{aligned}
$$

These integrals, and the ones below, are taken over all space and time. Furthermore, if $\delta>0$,

$$
\begin{aligned}
& \iint\left(|E|^{2}+|H|^{2}+|\Psi|^{2}\right) \frac{1}{(1+r)^{1+\delta}} d x d t<\infty, \\
& \iint|\phi|^{2} \frac{1}{(1+r)^{3+\delta}} d x d t<\infty .
\end{aligned}
$$

Proof. Estimates (40)-(42) follow immediately from the integrability of $Z$ in (39). Using the second term in (39) and shifting the origin $x=0$ to any other point, we obtain

$$
\pi \int_{-\infty}^{\infty}|\phi(x, t)|^{2} d t \leqq e_{0} \quad \text { for all } x
$$

Therefore

$$
\int\left[\int|\phi(x, t)|^{2} d t\right](1+r)^{-3-\delta} d x \leqq \pi^{-1} e_{0} \int(1+r)^{-3-\delta} d x<\infty .
$$

This proves (44). 
In order to prove (43) we choose a more general form for our multiplier, namely

$$
l_{j}(x)=\frac{x_{j}}{r} \zeta(r) .
$$

Then

$$
\begin{aligned}
\partial^{k} l_{j} & =\frac{\zeta}{r} \delta_{j k}-\left(\frac{\zeta}{r}-\zeta^{\prime}\right) x_{j} x_{k} / r^{2}, \\
m & =\partial^{j} l_{j}=\frac{2}{r} \zeta+\zeta^{\prime}, \\
m \delta_{j k}-2 \partial^{k} l_{j} & =\zeta^{\prime} \delta_{j k}+2\left(\frac{\zeta}{r}-\zeta^{\prime}\right) x_{j} x_{k} / r^{2} .
\end{aligned}
$$

The weight function $\zeta$ is chosen to satisfy the following constraints:
i) $\zeta$ bounded, $\frac{\zeta}{r} \geqq \zeta^{\prime} \geqq 0$
ii) $\partial^{k} \partial^{k} m=\frac{4}{r} \zeta^{\prime \prime}+\zeta^{\prime \prime \prime} \leqq 0$
iii) $l_{j} \partial^{j} m+\frac{1}{2} m^{2}=\frac{4}{r} \zeta \zeta^{\prime}+\frac{1}{2}\left(\zeta^{\prime}\right)^{2}+\zeta \zeta^{\prime \prime} \geqq 0$.

By (37) and (38) it follows that $\int|X| d x$ is bounded by a constant multiple of the energy $e_{0}$. The general expression for $Z$ implies that

$$
\begin{aligned}
Z \geqq & \zeta^{\prime}|E|^{2}+2\left(\frac{\zeta}{r}-\zeta^{\prime}\right)\left|E_{r}\right|^{2}+\zeta^{\prime}|H|^{2} \\
& +2\left(\frac{\zeta}{r}-\zeta^{\prime}\right)\left|H_{r}\right|^{2}+2 \frac{\zeta}{r}\left(|\Psi|^{2}-\left|\Psi_{r}\right|^{2}\right)+2 \zeta^{\prime}\left|\Psi_{r}\right|^{2} .
\end{aligned}
$$

Each of these six terms is non-negative and hence is integrable over all space-time.

Now we make the specific choice

$$
\zeta(r)=2-(r+1)^{-\delta}
$$

where $0<\delta<1$. We will verify (45). By explicit calculation, we have $\zeta^{\prime}=\delta(r+1)^{-1-\delta}$ and

$$
\begin{aligned}
\zeta / r-\zeta^{\prime}= & r^{-1}(r+1)^{-\delta}\left[2(r+1)^{\delta}-1+\delta r(r+1)^{-1}\right] \\
& >r^{-1}(r+1)^{-\delta}(1-\delta)>0 .
\end{aligned}
$$

Therefore (43) follows from (46). We need only complete the verification of (45). Indeed

$$
4 \zeta^{\prime \prime} / r+\zeta^{\prime \prime \prime}=-\delta(\delta+1) r^{-1}(r+1)^{-3-\delta}[(2-\delta) r+4]<0
$$

and

$$
\begin{aligned}
& 4 r^{-1} \zeta \zeta^{\prime}+\frac{1}{2}\left(\zeta^{\prime}\right)^{2}+\zeta \zeta^{\prime \prime} \geqq\left(4 r^{-1} \zeta^{\prime}+\zeta^{\prime \prime}\right) \zeta \\
& \quad=\zeta \delta r^{-1}(r+1)^{-2-\delta}[4+(3-\delta) r] \geqq 0 .
\end{aligned}
$$

This completes the proof of Theorem 4 . 
Corollary 3. If $\phi \cdot V^{\prime}(\phi) \geqq 2 V(\phi) \geqq 0$, there are no "classical lumps" of finite energy. That is, if $E(x), H(x), \phi(x)$ is a solution which is independent of time and has finite energy, then $E=H=\phi=0$.

In (43) we are missing an estimate on $\left|\psi^{0}\right|^{2}$. To get it we need a slightly stronger assumption on $V$, as follows.

Theorem 5. Assume that $V$ has the form

$$
V(\phi)=\frac{1}{2} m_{0}^{2}|\phi|^{2}+W(\phi),
$$

where

$$
0 \leqq \alpha W(\phi) \leqq \phi \cdot W^{\prime}(\phi) .
$$

We assume $m_{0}, \delta$, and $R$ are positive constants and $\alpha>2$. Then

$$
\begin{aligned}
& \iint W(\phi) \frac{1}{r} d x d t<\infty, \\
& \iint\left|\psi^{0}\right|^{2} \frac{1}{(r+1)^{3+\delta}} d x d t<\infty, \\
& \int_{-\infty}^{\infty} \int_{|x|<R} e d x d t<\infty, \\
& \int_{|x|<R} e d x \rightarrow 0 \text { as } \quad|t| \rightarrow \infty .
\end{aligned}
$$

Proof. We have

$$
\phi \cdot V^{\prime}(\phi)-2 V(\phi)=\phi \cdot W^{\prime}(\phi)-2 W(\phi) \geqq(\alpha-2) W(\phi) .
$$

Therefore (42) implies (47). Now we multiply identity (30) from the end of Sect. 3 by $(r+1)^{-3-\delta}=\xi(r)$. Thus

$$
\begin{aligned}
& \partial^{0}\left(\xi \psi^{0} \cdot \phi\right)+\xi\left(m_{0}^{2}|\phi|^{2}+\phi \cdot W^{\prime}(\phi)\right) . \\
& \quad=\xi\left(\left|\psi^{0}\right|^{2}-|\Psi|^{2}\right)+\partial^{k}\left(\xi \psi^{k} \cdot \phi\right)+(3+\delta)(r+1)^{-4-\delta_{r}-1} x_{k} \psi^{k} \cdot \phi .
\end{aligned}
$$

The last term is bounded by a constant times

$$
(r+1)^{-1-\delta}|\Psi|^{2}+(r+1)^{-3-\delta}|\phi|^{2},
$$

which is integrable over space-time. As for the first term in (51),

$$
\int \xi \psi^{0} \cdot \phi d x \leqq \frac{1}{2} \int\left(\left|\psi^{0}\right|^{2}+|\phi|^{2}\right) d x \leqq\left(1+m_{0}^{-2}\right) e_{0}
$$

because of the mass term in the energy. The terms $\xi|\Psi|^{2}, \xi|\phi|^{2}$ and $\xi \phi \cdot W^{\prime}(\phi)$ are also integrable over space-time because

$$
\phi \cdot W^{\prime}(\phi)=\left[\phi \cdot V^{\prime}(\phi)-2 V(\phi)\right]+2 W(\phi) .
$$

Hence (51) implies that $\xi\left|\psi^{0}\right|^{2}$ is also integrable. This is (48). Since

$$
2 e=|E|^{2}+|H|^{2}+|\Psi|^{2}+\left|\psi^{0}\right|^{2}+m_{0}^{2}|\phi|^{2}+2 W(\phi),
$$

(49) follows from (43), (44), (47), and (48) as soon as we replace the factor $r+1$ in the denominators by the constant $R+1$. 
We now derive (50) from (49) using the method of Morawetz [2]. Let

$$
f(t)=\int_{R}^{R+1} \int_{|x|<\varrho} e d x d \varrho \geqq \int_{|x|<R} e d x .
$$

We will show that $f(t) \rightarrow 0$ as $|t| \rightarrow \infty$. By (49), $f(t)$ is integrable. Therefore it is enough to show that the derivative $f^{\prime}(t)$ is bounded $(-\infty<t<\infty)$.

Now

$$
\begin{aligned}
f^{\prime}(t) & =\int_{R}^{R+1} \int_{|x|<\varrho} \partial^{0} e d x d \varrho \\
& =\int_{R}^{R+1} \int_{|x|=\varrho}\left(\sum_{k} \frac{x_{k}}{r} p^{k}\right) d S_{x} d \varrho \quad \text { by }(20) \\
& =\int_{R<|x|<R+1}\left(\sum_{k} \frac{x_{k}}{r} p^{k}\right) d x .
\end{aligned}
$$

Thus $\left|f^{\prime}(t)\right| \leqq \int_{R<|x|<R+1} e d x \leqq \int e d x=e_{0}$.

This completes the proof of Theorem 5 .

Finally, we establish the square integrability of the potentials $A^{\mu}$ themselves. We first assume only that $V(\phi) \geqq 0$. It follows as in [1] that

$$
\begin{aligned}
\left(\sum_{\mu} \int\left|A^{\mu}(x, t)\right|^{2} d x\right)^{1 / 2} \leqq & \left(\sum_{\mu} \int\left|A^{\mu}(x, 0)\right|^{2} d x\right)^{1 / 2} \\
& +\int_{0}^{t}\left(\int|E(x, s)|^{2} d x\right)^{1 / 2} d s
\end{aligned}
$$

in, say, the Lorentz gauge. This comes from multiplying (6) by $A^{k}$, summing over $k=1,2,3$, and integrating. In particular;

$$
\int \sum_{\mu}\left|A^{\mu}(x, t)\right|^{2} d x=O\left(1+t^{2}\right)
$$

for all $t$. We can also estimate $\int|\phi|^{2} d x$, even in the case of zero mass. We integrate the identity

$$
\phi \cdot \psi^{0}=\phi \cdot D^{0} \phi=\frac{1}{2} \partial^{0}\left(|\phi|^{2}\right)
$$

to obtain

$$
\partial^{0} \int|\phi|^{2} d x \leqq 2\left(\int|\phi|^{2} d x\right)^{1 / 2}\left(\int\left|\psi^{0}\right|^{2} d x\right)^{1 / 2} .
$$

Therefore

$$
\begin{aligned}
\left(\int|\phi(x, t)|^{2} d x\right)^{1 / 2} \leqq & \left(\int|\phi(x, 0)|^{2} d x\right)^{1 / 2} \\
& +\int_{0}^{t}\left(\int\left|\psi^{0}(x, s)\right|^{2} d x\right)^{1 / 2} d s .
\end{aligned}
$$

Since $V(\phi) \geqq 0$, we conclude that

$$
\int|\phi(x, t)|^{2} d x=O\left(1+t^{2}\right) \text { for all } t .
$$


We can find stronger bounds on $\int|\phi|^{2} d x$ if we assume that $\varphi \cdot V^{\prime}(\phi) \geqq 4 V(\phi) \geqq 0$ and that $\int r^{2} e d x<\infty$. For then, we rewrite the integrated First Inversional Law (27) in the form:

$$
\begin{aligned}
& \frac{1}{2} \int\left(r^{2}+t^{2}\right)\left(|E|^{2}+|H|^{2}\right) d x+2 t \int r \sum_{k} \omega_{k} p_{Y M}^{k} d x+\int\left(t^{2}+r^{2}\right) V(\phi) d x \\
& \quad+\frac{1}{2} \int r^{2}\left(|\Psi|^{2}-\left|\Psi_{r}\right|^{2}\right) d x+t \int \varphi \cdot \psi^{0} d x \\
& \quad+\frac{1}{2} \sum_{k} \int\left|t \psi^{k}+x_{k} \psi^{0}\right|^{2} d x+\frac{1}{2} \int\left|\Psi x+t \psi^{0}+\phi\right|^{2} d x \leqq C .
\end{aligned}
$$

It follows that

$$
t \int \phi \cdot \psi^{0} d x \leqq C
$$

so that (52) yields

$$
\frac{1}{2} \int \partial^{0}\left(|\phi|^{2}\right) d x \leqq \frac{C}{t} \text { for } t \geqq 1, \text { say. }
$$

Hence $\int|\phi(x, t)|^{2} d x=O(\ln t)$ as $t \rightarrow \infty$.

Acknowledgement. We are grateful to A. Jaffe for suggesting that we try the methods of [1] on this system.

\section{References}

1. Glassey, R.T., Strauss, W.A.: Decay of classical Yang-Mills fields. Commun. Math. Phys. 65, 1-13 (1979)

2. Morawetz, C.S. : Time decay for the nonlinear Klein-Gordon equation. Proc. R. Soc. A306, 291-296 (1968)

3. Strauss, W.A.: Nonlinear invariant wave equations. In: Lecture notes in physics, Vol. 73, pp. 197-249. Berlin, Heidelberg, New York: Springer 1978

Communicated by A. Jaffe

Received November 7, 1978 
